\title{
A importância da determinação da hemoglobina glicada no monitoramento das complicações crônicas do diabetes mellitus
}

The importance of glycated hemoglobin determination in the management of chronic complications associated with diabetes mellitus

Andreza Fabro de Bem'; Juliana Kunde²

\begin{tabular}{|c|c|}
\hline unitermos & resuno \\
\hline Diabetes mellitus & O diabetes é uma situação clínica muito freqüente que envolve cerca de $7 \%$ da população mundial. Por \\
\hline Hemoglobina glicada & $\begin{array}{l}\text { essa razão muitos esforços têm sido empregados na implementação de métodos de monitoramento } \\
\text { e no desenvolvimento de terapias efetivas para o seu controle. A hemoglobina qlicada }(\mathrm{HbA} 1 \mathrm{c}) \text { é o }\end{array}$ \\
\hline & teste mais indicado na quantificação do risco de complicações crônicas em pacientes diabéticos. O \\
\hline Complicaçōes crônicas & $\begin{array}{l}\text { Diabetes Control and Complications Trial (DCCT) e o United Kingdom Prospective Diabetes Study } \\
\text { (UKPDS) concluíram que o risco de complicações em pacientes diabéticos é diretamente proporcional } \\
\text { ao controle glicêmico, determinado através dos níveis de HbA1c. A medida exata e precisa da HbA1c } \\
\text { é uma questão importante para os laboratórios clínicos, sendo que vários fatores podem afetar as } \\
\text { determinações, levando a resultados equivocados. O objetivo deste estudo é demonstrar os diferentes } \\
\text { métodos para a quantificação da HbA1c, bem como discutir os problemas mais freqüentes de padro- } \\
\text { nização dessa determinação. }\end{array}$ \\
\hline
\end{tabular}

\section{abstract}

Diabetes is a widespread disease, involving about $7 \%$ of the entire world population. For this reason, many efforts have been devoted to the wide application of valid monitoring procedures and to the development of effective therapeutic approaches. Glycated hemoglobin $(\mathrm{HbA1c})$ is the pre-eminent factor for quantifying the risk of chronic complications in patients with diabetes. The Diabetes Control and Complications Trial (DCCT) and United Kingdom Prospective Diabetes Study (UKPDS), demonstrated conclusively that risks for complications in patients with diabetes are directly related to glycemic control, as measured by glycated hemoglobin ( $\mathrm{HbA1c}$ ). Accurate determination of $\mathrm{HbA1} \mathrm{c}$ is an important issue for clinical laboratories and several factors may affect and lead to erroneous results. The main objective of this study is to show the different methods for glycated hemoglobin quantification and to discuss the most frequent problems of standardization glycated hemoglobin measurements. key words

Diabetes mellitus

Glycated hemoglobin

HbA1C

Chronic complications

\section{Introdução}

O diabetes mellitus (DM) é uma síndrome de etiologia múltipla decorrente da falta de insulina e/ou da incapacidade da insulina em exercer adequadamente seus efeitos ${ }^{(19)}$. É uma situação clínica freqüente que acomete cerca de $7,6 \%$ da população adulta entre 30 e 69 anos $^{(16)}$ e 0,3\% das gestantes ${ }^{(2)}$.
Em 1997, a Associação Americana de Diabetes (ADA) apresentou novas recomendações sobre o diagnóstico e classificação do $\mathrm{DM}^{(22)}$, as quais são revisadas anualmente ${ }^{(3)}$. O método diagnóstico recomendado é a dosagem de glicemia em jejum e o teste de tolerância à glicose (TTG) em algumas circunstâncias. Em consenso recente da ADA, o limite máximo da normalidade da glicemia

1. Professora de Bioquímica Clínica da Universidade Federal de Santa Maria (UFSM).

2. Farmacêutica Bioquímica do Laboratório Fischer; aluna do Curso de Especialização em Laboratório Clínico do Departamento de Análises Clínicas e Toxicológicas da UFSM. 
de jejum passou a ser de $99 \mathrm{mg} / \mathrm{dl}$, sendo que a glicemia de jejum inapropriada (pré-diabetes) está definida entre 100 e $125 \mathrm{mg} / \mathrm{dl}^{(10)}$.

As conseqüências do DM a longo prazo acontecem devido a alterações micro e macrovasculares que levam a disfunção, dano ou falência de vários órgãos. As complicações crônicas compreendem a nefropatia, com possível evolução para insuficiência renal, a retinopatia, com possibilidade de cegueira, e a neuropatia, com risco de úlceras nos pés, amputações, artropatia de Charcot e manifestações de disfunção autonômica, incluindo disfunção sexual. Pessoas com diabetes apresentam elevado risco de doença vascular aterosclerótica, como as doenças coronariana, arterial periférica e vascular cerebral(19).

A dosagem da glicose no sangue não constitui parâmetro eficiente para avaliação do controle da glicemia durante um intervalo de tempo prolongado ${ }^{(8)}$. Nesse sentido, a dosagem da hemoglobina glicada ( $\mathrm{HbA1c}$ ) tem papel fundamental na monitorização do controle glicêmico em pacientes diabéticos, pois fornece informações acerca do índice retrospectivo da glicose plasmática ${ }^{(7,8,21,24,29)}$. A grande vantagem da $\mathrm{HbA} 1 \mathrm{c}$ está no fato de não sofrer grandes flutuações, como na dosagem da glicose plasmática, bem como estar diretamente relacionada ao risco de complicações em pacientes com DM tipos 1 e $2^{(8)}$.

Do ponto de vista metodológico, a dosagem de $\mathrm{HbA} 1 \mathrm{c}$ evoluiu consideravelmente desde o início de seu uso em rotina diagnóstica. No princípio, os métodos disponíveis baseavam-se em cromatografia de troca iônica, que apresentava como inconvenientes grande dependência da temperatura ambiente e da qualidade dos tampões, e interferência de hemoglobinas anormais ( $S, C$, etc.). A seguir desenvolveram-se métodos mais estáveis, baseados na cromatografia de afinidade, que apresentavam, entretanto, dificuldades de automação e uso intensivo de mão-de-obra, o que ocasiona, em conseqüência, uma reprodutibilidade longe da ideal ${ }^{(30)}$. Uma dosagem precisa e reprodutível da HbA1c é de fundamental importância, principalmente quando se pretende usá-la como base para variações em regimes de terapia intensiva, e tem sido uma preocupação constante descrita na literatura recente $\mathrm{e}^{(9,24,30)}$.

\section{Complicações do diabetes mellitus}

As complicações crônicas do DM são as principais responsáveis pela morbidade e mortalidade dos pacientes diabéticos. As doenças cardiovasculares representam a principal causa de morte (52\%) em pacientes diabéticos do tipo $2^{(18)}$.
A estratégia de prevenção das complicações crônicas do diabetes baseia-se no controle da hiperglicemia para tratamento precoce de suas complicações. É consenso a necessidade da manutenção de um controle glicêmico satisfatório em todos os pacientes, isto é, um grau de controle que previna a sintomatologia aguda e crônica atribuída à hiperglicemia e à hipoglicemia ${ }^{(6)}$.

No DM1 este controle glicêmico satisfatório é capaz de prevenir/retardar complicações microvasculares e neuropáticas, como demonstrado pelos dados do Diabetes Control and Complications Trial (DCCT) $)^{(8)}$. Este estudo acompanhou 1.441 indivíduos com DM1 randomizados em dois grupos: um com tratamento insulínico intensivo (três ou mais injeções diárias de insulina) e outro em tratamento convencional (uma a duas injeções de insulina por dia). Após 6,5 anos o estudo demonstrou que o tratamento intensivo viabiliza um controle satisfatório com níveis médios de $\mathrm{HbA} 1 \mathrm{c}$ de 7\%, prevenindo o aparecimento e reduzindo a progressão de nefropatia, retinopatia e neuropatia diabéticas, quando em comparação com o controle obtido pelo tratamento convencional, no qual os níveis de $\mathrm{HbA1c}$ são, em média, 9\%.

A nefropatia está presente em $15 \%$ a $20 \%$ dos pacientes com DM2 e em $30 \%$ a $40 \%$ dos diabéticos tipo 1 com longa evolução. Trata-se da principal causa de insuficiência renal em pacientes que fazem diálise ${ }^{(20)}$. Os dados combinados do DCCT demonstram que o controle glicêmico satisfatório do DM1 pode reduzir a incidência de microalbuminúria em $39 \%$ e de albuminúria em $54 \%{ }^{(8)}$. A retinopatia diabética acomete cerca de $40 \%$ dos pacientes diabéticos e é a principal causa de cegueira em pacientes entre 25 e 74 anos $^{(1)}$. O DCCT mostrou que o controle satisfatório da glicemia pode reduzir a incidência de retinopatia em $76 \%$ e sua taxa de progressão em $54 \%{ }^{(8)}$. A neuropatia diabética é a complicação mais freqüente e precoce do $\mathrm{DM}$, podendo atingir $80 \%$ a $100 \%$ dos pacientes a longo prazo e sendo retardada pelo controle glicêmico rigoroso. O pé diabético é a principal lesão de extremidade, sendo assim denominado devido às lesões dos pés decorrentes da neuropatia e, principalmente, da doença vascular periférica. Pacientes diabéticos têm em torno de 15 vezes maior risco de sofrer amputações que os não-diabéticos e $20 \%$ dos amputados morrem em dois anos ${ }^{(20)}$.

A glicação não-enzimática de proteínas ou reação de Maillard é um processo ligado à hiperglicemia crônica, a qual, por sua vez, ocasiona uma série de alterações fisiológicas importantes no desenvolvimento das complicações crônicas do diabetes ${ }^{(5)}$. Como pode ser observado na 
Figura 1, a reação de Maillard é subdivida em três estágios: inicial, intermediário e tardio. No estágio inicial, a glicose (ou outros açúcares redutores como frutose, galactose, manose e xilose) reage com grupamentos amino livres de várias moléculas, incluindo proteínas, ácidos nucléicos e lípides, formando um composto instável chamado base de Schiff. Esta molécula sofre um rearranjo, produzindo uma cetoamina estável, o produto de Amadori. Como essa reação não requer a participação de enzimas, as variáveis que regulam os níveis dos produtos glicosilados in vivo são: a concentração de glicose e proteína, a meia-vida da proteína e sua reatividade com os grupamentos amino. No estado intermediário, através de reações de oxidação e deidração, os produtos de Amadori são degradados em compostos carbonil (glioxal, metilglioxal e deoxiglicosona), os quais são muito mais reativos que os açúcares dos quais foram originados, agindo como propagadores de reações com grupamentos amino de várias proteínas, originando de forma irreversível compostos insolúveis, freqüentemente fluorescentes, usualmente chamados produtos finais de glicação avançada (AGEs). Estes se acumulam no organismo, sendo responsáveis pelas complicações crônicas do diabetes ${ }^{(14)}$. Os três mecanismos pelos quais os AGEs causam dano tecidual são ligação a macromoléculas, interação com receptores específicos e acumulação intracelular ${ }^{(27)}$.

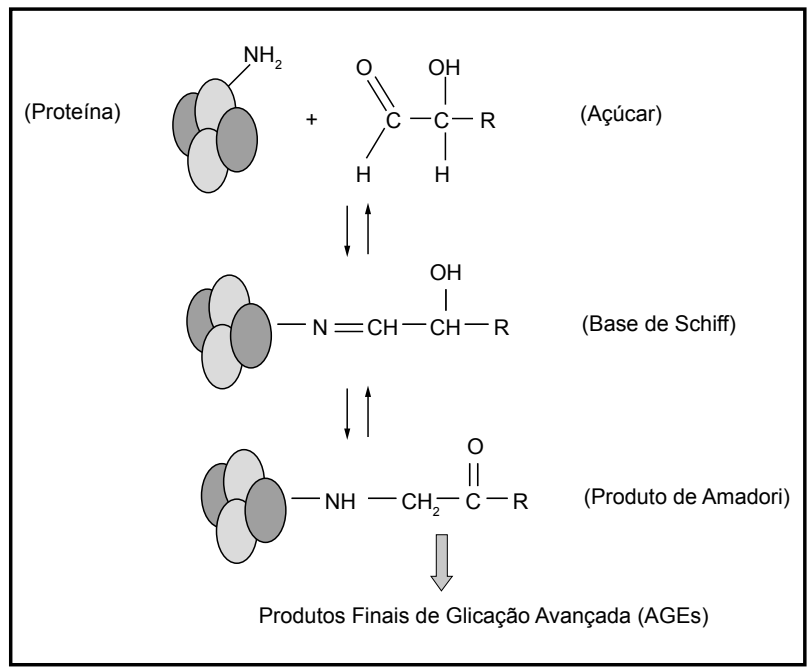

Figura 1 - Estágios da glicação não-enzimática das proteínas (adaptado de Lapolla et al., 2004)

\section{Hemoglobina glicada}

A determinação dos produtos de glicação (produtos de Amadori) é rotineiramente analisada através da dosagem da HbA1c, a qual é utilizada para avaliação do controle metabólico nos pacientes diabéticos.
A HbA1c origina-se por meio de uma reação irreversível entre a glicose sangüínea e o aminoácido valina $\mathrm{N}$-terminal da cadeia beta da hemoglobina A. A HbA1c representa aproximadamente $80 \%$ da fração das hemoglobinas $A_{1}$, também chamadas de rápidas, sendo esta denominação resultado do processo de separação eletroforética ${ }^{(25)}$.

Em um indivíduo não-diabético, cerca de $4 \%$ a $6 \%$ do total de $\mathrm{HbA} 1 \mathrm{C}$ apresenta-se glicada, enquanto que no diabético com descontrole acentuado esta porcentagem pode atingir níveis duas a três vezes acima do normal(25). Níveis de $\mathrm{HbA1c}$ acima de $7 \%$ estão associados a um risco progressivamente maior de complicações crônicas. Por isso, o conceito atual de tratamento do diabetes por objetivos define $7 \%$ como o limite superior acima do qual está indicada a revisão do esquema terapêutico em vigor ${ }^{(4,16)}$.

As outras frações da hemoglobina $A_{1}$ originam-se da ligação de outros elementos à porção valina $\mathrm{N}$-terminal da cadeia beta da hemoglobina $A: A_{1 a 1}$ (frutose 1,6-bifosfato), $A_{1 \mathrm{az}}$ (frutose 6-fosfato) e $A_{1 \mathrm{~b}}$ (ácido pirúvico). Quando o processo de glicação ocorre em outros pontos da cadeia beta e na cadeia alfa, origina-se a molécula de hemoglobina glicada $\mathrm{A}_{0}$. O somatório de todas as frações da hemoglobina $A_{1}$ e $A_{0}$ resulta na chamada hemoglobina glicada total ${ }^{(25)}$.

No processo de gênese da HbA1c, observa-se a formação de uma base de Schiff denominada também de aldimina ou pré-A1c. O ritmo de formação desse composto é diretamente proporcional à concentração de glicose plasmática, sendo que esta molécula pode dissociar-se ou formar uma cetoamina estável, não mais dissociável, agora denominada de $\mathrm{HbA} 1 \mathrm{c}^{(25)}$.

Alguns métodos disponíveis no mercado para dosagem da hemoglobina glicada conseguem separar a fração HbA1c das outras moléculas análogas, ou seja, a base de Schiff, a hemoglobina $\mathrm{A}_{0}$ e as outras frações da hemoglobina $\mathrm{A}_{1}$. Os métodos que não conseguem caracterizar esses elementos podem, potencialmente, gerar valores não-compatíveis com o real estado de controle dos níveis glicêmicos ${ }^{(12)}$.

O nível de hemoglobina glicada é resultado de todas as hemácias circulantes no organismo, desde a mais velha (120 dias) à mais jovem. Porém a glicose dos últimos 30 dias antes da dosagem da hemoglobina glicada contribui com praticamente $50 \%$ da $\mathrm{HbA} 1 \mathrm{c}$, enquanto os níveis glicêmicos dos últimos dois a quatro meses contribuem com aproximadamente $25 \%$. Conclui-se, desta forma, que a hemoglobina glicada reflete, na realidade, a média ponderada do níveis glicêmicos de 60 a 90 dias antes do exame $^{(7,24,25,32) \text {. }}$. 


\section{Metodologias para quantificação} da hemoglobina glicada

Embora mais de 20 procedimentos diferentes estejam disponíveis para a quantificação da $\mathrm{HbA} 1 \mathrm{c}^{(13)}$ em termos de rotina laboratorial, podemos dividir estas metodologias em dois grandes blocos, segundo o princípio utilizado na separação da fração glicada da não-glicada.

Como pode ser observado na Tabela 1, dependendo da metodologia empregada, o resultado poderá refletir, por exemplo, o total de hemoglobina glicada ou apenas uma das frações glicadas, como a HbA1c. Essas diferenças são muito importantes no momento da interpretação dos resultados, bem como na identificação de possíveis interferentes metodológicos.

Na cromatografia por troca iônica, a hemoglobina não-glicada apresenta uma carga positiva, ajustando-se o $\mathrm{pH}$ do meio reacional, quando comparada à hemoglobina glicada, o que a faz interagir mais com uma coluna catiônica (carga negativa). O fluxo de um tampão adequado na resina permite eluir a fração glicada, portanto separando-a da não-glicada pela carga da molécula de hemoglobina ${ }^{(11)}$. Essa metodologia está disponível em minicolunas cromatográficas ou em sofisticados sistemas de high performance liquid chromatography (HPLC), os quais podem apresentar-se completamente automatizados.

A cromatografia de afinidade utiliza derivados do ácido borônico, como o ácido m-aminofenilborônico, imobilizados em uma resina. $\mathrm{O}$ ácido borônico reage com cis dióis (compostos que apresentam duas hidroxilas no mesmo lado, como a molécula de glicose), portanto a separação das frações glicada e não-glicada se dá pela porção açúcar, ficando a hemoglobina glicada retida na coluna enquanto a não-glicada (ou $\mathrm{HbA}_{0}$ ) é eluída da mesma pelo fluxo de um tampão. Este princípio metodológico quantifica primariamente a hemoglobina glicada total ${ }^{(17)}$.

As referidas características entre os princípios devem ser analisadas com cautela quando se pretende caracterizar vantagens e desvantagens dos referidos procedimentos. Os sistemas automatizados e semi-automatizados disponíveis possibilitam aos dois processos performances semelhantes em termos de imprecisão (reprodutibilidade medida pelo coeficiente de variação) e sensibilidade, eliminando também os principais problemas do sistema de troca iônica, como a influência da temperatura ambiente sobre a capacidade de ligação da resina e a interferência da fração lábil (28).

A Tabela 2 compara as principais características dos sistemas cromatográficos de troca iônica e de afinidade. Os dois princípios metodológicos são diferentes e, portanto, separam populações de hemoglobinas glicadas diferentes. Na troca iônica, a separação ocorre pela carga da molécula de hemoglobina, portanto patologias que alteram a carga da hemoglobina poderão afetar sua interação com a resina e produzir resultados falsos. Por exemplo, hemoglobinopatias, carbamilação da hemoglobina, ou, ainda, a possibilidade de drogas (como o ácido acetilsalićlico em altas doses) que podem ligar-se à hemoglobina, alterando-lhe a carga. $\mathrm{Na}$ cromatografia de afinidade, a separação, ocorrendo pela fração glicada, não sofre influência das hemoglobinas variantes ou de outras alterações na estrutura protéica. No entanto, esse processo não quantifica uma fração, mas sim o conjunto total das hemoglobinas glicadas.

Os resultados para hemoglobina glicada obtidos pelos métodos de cromatografia de troca iônica e afinidade, embora altamente correlacionados, não são iguais, sendo

\section{Tabela 1 Principais métodos para quantificar a hemoglobina glicada}

\begin{tabular}{ccc}
\hline Principio & Métodos & Componente medido \\
\hline Separação pela diferença na carga & HPLC (troca iônica) & HbA1c \\
& Troca iônica (coluna) & HbA1c \\
& & HbA $_{1}$ \\
Separação pela diferença estrutural & Eletroforese & HbA1c \\
& & HbA $_{1}$ \\
& HPLC (afinidade) & Hb glicada total \\
& Afinidade (coluna) & Hb glicada total \\
\hline
\end{tabular}

Adaptado de Sacks, 1999. 
Tabela 2

Comparação entre as principais características dos sistemas cromatográficos de troca iônica e

Caracteristicas

Utilidade clínica

Fração quantificada

Interferência da fração lábil (pré- $\mathrm{A}_{1 c}$ )

Suscetibilidade do ensaio a variação

de temperatura

Interferência por hemoglobinas

variantes (com alteração na carga,

como $\mathrm{Hb} \mathrm{S}$ e C)

Interferência por hemoglobina car-

bamilada, outras drogas que alterem

a carga da hemoglobina

Possibilidade de automação

Adaptado de Turpenein, 1995.

essa desigualdade muito mais evidente nos pacientes com diabetes mal controlado, quando a glicemia média sangüínea é maior.

\section{Principais interferências na determinação da hemoglobina glicada}

Patologias que alterem a vida média da hemácia (e conseqüentemente da hemoglobina) alteram os resultados de hemoglobina glicada realizados por qualquer metodologia. Portanto, este ensaio requer, para sua plena interpretação, que as hemácias apresentem uma vida média normal. As doenças que cursam com anemia hemolítica ou estados hemorrágicos podem resultar em valores inapropriadamente diminuídos por encurtarem a sobrevida das hemácias ${ }^{(24,26,32)}$; patologias que aumentam a vida média da hemácia, como as anemias por carência de ferro, vitamina B12 ou folatos, podem resultar em valores inapropriadamente elevados por aumentar a sobrevida das hemácias ${ }^{(24,26,32)}$.

A dosagem de hemoglobina glicada em pacientes portadores de hemoglobina variante heterozigótica (exemplos: hemoglobinas S, C, Graz, Sherwood, Forest, D, Padoya) resulta em valores falsamente elevados ou rebaixados, conforme a metodologia aplicada. O método HPLC pode identificar a presença de hemoglobina anômala, permitindo uma análise mais crítica do resultado obtido; já os métodos de imunoensaio não são capazes de detectar a presença de hemoglobinas variantes ${ }^{(7,12,23)}$.
A quantificação da hemoglobina glicada não é aplicável nas hemoglobinopatias homozigóticas, independente da metodologia utilizada, em função da ausência de hemoglobina A. Essa condição necessita ser rastreada e confirmada pelos métodos usuais para o estudo das hemoglobinopatias. Nessas situações, exames alternativos, como a frutosamina e a albumina glicada, podem ser úteis ${ }^{(7,12)}$.

Hipertrigliceridemia, hiperbilirrubinemia, uremia, alcoolismo crônico, ingestão crônica de salicilatos e opiáceos podem interferir em algumas metodologias, produzindo resultados falsamente elevados ${ }^{(7,20,24)}$. Em pacientes com nefropatias crônicas, os resultados da hemoglobina glicada devem ser avaliados com cautela. Se medida como HbA1c por troca iônica, o resultado pode sofrer interferência da carbamilação da hemoglobina devido à uremia intensa, independente do princípio metodológico pela anemia quase sempre presente nestas patologias ${ }^{(31)}$.

A presença de grandes quantidades de vitaminas $C$ e Eé descrita como fator que pode induzir resultados falsamente diminuídos por inibirem a glicação da hemoglobina ${ }^{(24)}$.

\section{Pontos importantes a ressaltar no uso clínico da hemoglobina glicada}

A determinação dos níveis da HbA1c é a melhor opção para a avaliação do controle glicêmico em médio e longo prazos. Entretanto, esse processo não é indicado para o diagnóstico do $\mathrm{DM}^{(3,24)}$. 
A hemoglobina glicada deve ser medida rotineiramente em todos os pacientes com DM para documentar o grau de controle glicêmico. As metas de tratamento devem ser baseadas em resultados de estudos clínicos prospectivos e randomizados, como DCCT e o United Kingdom Prospective Diabetes Study (UKPDS). Esses estudos mostraram uma correlação entre o controle glicêmico, quantificado por determinações seriadas de $\mathrm{HbA} 1 \mathrm{c}$, e os riscos de desenvolvimento e progressão das complicações crônicas do diabetes ${ }^{(8,24,29)}$.

Os testes de HbA1c devem ser realizados pelo menos duas vezes ao ano por todos os pacientes diabéticos e quatro vezes por ano (a cada três meses) por pacientes que se submeterem a alterações do esquema terapêutico ou que não estejam atingindo os objetivos recomendados com o tratamento vigente ${ }^{(24)}$.

Níveis de $\mathrm{HbA} 1$ c acima de 7\% estão associados a risco progressivamente maior de complicações crônicas. Por isso o conceito atual de tratamento do diabetes por objetivos define em $7 \%$ o limite superior acima do qual está indicada a revisão do esquema terapêutico em vigor ${ }^{(8,29)}$.

\section{Considerações finais}

Os estudos prospectivos de intervenção, DCCT e UKPDS, demonstraram de forma inequívoca que a manutenção de taxas glicêmicas em valores próximos do normal avaliadas pelo teste de hemoglobina glicada é acompanhada de redução significativa do surgimento e da progressão das complicações microvasculares, tanto em diabéticos tipo 1 (DCCT) $)^{(19)}$, quanto tipo 2 (UKPDS) $)^{(16)}$. Nesse sentido, o teste de $\mathrm{HbA} 1 \mathrm{c}$ é fundamental no acompanhamento dos diabéticos, sendo que o resultado encontrado é determi- nante na conduta médica adotada para estes indivíduos. É o exame mais informativo disponível no momento em relação à prevenção de complicações crônicas e no controle do diabetes mellitus.

Os métodos baseados em HPLC apresentam o potencial de automação e a reprodutibilidade desejáveis. Em função disso, o DCCT ${ }^{(8)}$ adotou o HPLC como o método de referência interino até haver algum definitivo, o qual demonstre excelente precisão, boa reprodutibilidade, baixa influência nos resultados da presença de hemoglobinas anormais, bem como da possibilidade de total automatização. No Brasil, o Posicionamento Ofical 2004(32) recomenda que os laboratórios clínicos dêem preferência aos métodos rastreáveis ao DCCT, conforme certificado do National Glycohemoglobin Standardization Program (NGSP), uma vez que não existe ainda programa semelhante no país. Além disso, o laboratório deve participar ativa e regularmente de um programa de ensaios de proficiência específico.

Como está evidente que a quantificação da hemoglobina glicada é dependente da metodologia empregada, é recomendável que os controles do mesmo paciente diabético sejam realizados sempre no mesmo laboratório ou pelo menos em laboratórios que apresentem metodologias semelhantes. Devido ao grande número de fatores que podem afetar os diferentes métodos, cada laboratório deve estar atento às fontes de erros que podem alterar seus resultados. Embora a maioria dessas interferências esteja relatada na literatura internacional, os resultados dos estudos são contraditórios ${ }^{(33)}$. Os laboratórios clínicos devem estar preparados para fornecer resultados exatos e precisos na determinação da $\mathrm{HbA1c}$, para ajudar o clínico na prevenção das complicações crônicas do DM.

\section{Referências}

I.AIELLO, L. P. et al. Diabetic retinopathy.Technical review. Diabetes Care, v. 2 I, p. 143-56, 1998.

2. AMERICAN DIABETES ASSOCIATION. Gestational diabetes mellitus. Clinical Practice Recommendations 200 I. Diabetes Care, v. 24, suppl. I, p. S77-9, 2001.

3. AMERICAN DIABETES ASSOCIATION. Clinical Practice Recommendations. Report of the Expert Committee on the Diagnosis and Classification of Diabetes Mellitus. Diabetes Care, v. 26, suppl. I, p. S5-20, 2003.

4. AMERICAN DIABETES ASSOCIATION. Position Statement. Standards of Medical Care for Patients with Diabetes mallitus. Diabetes Care, v. 22, p. S32-S4I, 1999.
5. BROWNLEE, M.; CERAMI, A.; VLASSARA, H. Advanced glycosylation end products and the biochemical basis of diabetic complications. N Engl J Med, v. 318, p. |3|5-21, 1988.

6. DUNCAN, B.; SCHIMDT, M. I.; GIULIANI, E. R. J. Medicina ambulatorial: condutas clínicas em atenção primária. 2. ed. Porto Alegre: Artemed, 1996.

7. BRY, L.; CHEN, P. C.; SACKS, D. B. Effects of hemoglobin variants and chemically modified derivates on assay for glycohemoglobin. Clin Chem, v. 47, p. 153-63, 2001.

8.DCCT RESEARCH GROUP.Diabetes Control and Complications Trial (DCCT).The effect of intensive treatment of Diabetes 
on the development and progression of the long-term complications in insulin-dependent diabetes mellitus. N Engl J Med, v. 329, p. 977-86, 1993.

9. ECKFELDT, J. H.; BRUNS, D. E. Another step towards standardization of methods for measuring hemoglobin A I c. Clin Chem, v. 43, p. I81 |-3, 1997.

10. FOLLOW-UP Report on the Diagnosis of Diabetes Mellitus. The Expert Committee on the Diagnosis and Classification of Diabetes Mellitus. Diabetes Care, v. 26, p. 3160-7, 2003.

1 I. HALWACHS-BAUMANN, G. et al. Comparative evaluation of three assay systems for automated determination of hemoglobin A I c. Clin Chem, v. 43, p. 5I I-7, 1997.

12. KHUU, H. M. et al. Evaluation of a fully automated highperformance liquid chromatography assay for hemoglobina A I c. Arch Pathol Lab Med, v. I23, p. 763-7, 1999.

13. KOBOLD, U. et al. Candidate reference methods for hemoglobin Alc based on peptide mapping. Clin Chem, v. 43, p. 1944 5I, 1997.

14. LAPOLLA, A. et al. Enzymatic digestion and mass spectrometry in the study of advanced glycation and products/peptides. Am Mass Spectrom, v. I5, p. 496-509, 2004.

15. LIST of NGSP certified methods (update $7 / 03$, listed by date certified) (on line). Disponível em: <http://www.missouri. edu/ diabetes/ngsp.html>. Acesso em 25 jul 2003.

16. MALERBI, D.; FRANCO, L. Multicenter study of the prevalence of diabetes mellitus and impaired glucose tolerance in the urban Brazilian population aged 30-69 Yr. Diabetes Care, v. 15, p. 1509-16, 1992.

17. MALLIA, A. et al. Preparation na use of s boronic acid affinity support for separation and quantitation of glycosylated hemoglobins. Analytical Letters, v. 14, p. 649-61, 1981.

I8. NATHAN, D. M.; MEIGS, J.; SINGER, D. E.The epidemiology of cardiovascular disease in type 2 diabetes mellitus: how sweet is it? The Lancet, v. 350, suppl. I, p. 4-9, 1997.

19. OLIVEIRA, J. E. P. Consenso brasileiro sobre diabetes 2002: diagnóstico e classificação do diabetes melito e tratamento do diabetes melito do tipo 2. Sociedade Brasileira de Diabetes. Rio de Janeiro: Diagraphic, 2003. p. 72.

20. PAZ, G. et al. Liga Acadêmica de Diabetes, Universidade Federal do Paraná, 1999. Disponível em: <http://www.
psfmonteverde.hpg.ig.com.br/di_diabtes.html>.Acesso em 27 jun 2004.

21. PETERSON, K. P. et al. What is hemoglobin $A_{1}$ c? An analysis of glycated hemoglobins by electropray ionization mass spectrometry. Clin Chem, v. 44, p. 1951-8, 1998.

22. REPORT of the Expert Committee on the Diagnosis and Classification of Diabetes Mellitus. Diabetes Care, v. 20, p. I | 83-997, 1997.

23. ROBERTS, W. et al. Glycohemoglobin results in sample with hemoglobin $\mathrm{C}$ or $\mathrm{S}$ trait: comparison or four test systems. Clin Chem, v. 45, p. 906-9, 1999.

24. SACKS, D. B. et al. Guidelines and recomendations for laboratopry analysis in the diagnosis and management of diabetes mellitus. Clin Chem, v. 48, p. 436-72, 2002.

25. SACKS, D.B. Carbohydrate. In: BURTIS, C. A; ASHWOOD, E. R. (eds.) Tietz Textbook of Clinical Chemistry. Philadelphia:W.B. Saunders Company, 1999. p.750-808.

26. SACKS, D. B. Hemoglobin variants and hemoglobin A I c analysis: problem solved? Clin Chem, v. 49, p. 1245-7, 2003.

27. SING, A.; BARDEN, A. M.T.; BEILIN, L. Advanced glycation end products: a review. Diabetologia, v. 44, p. 129-46, 2001.

28. TURPEINEN, U. et al. Three assays for glycohemoglobin compared. Clin Chem, v. 4I, p. 191-5, 1995.

29. UK PROSPECTIVE DIABETES STUDY GROUP. Intensive blood glucose control with sulphonylureas or insulin compared with conventional treatment and risk of complications in the patients with type 2 Diabetes. Lancet, v. 352, p. 837-53, 1998.

30. WEYKAMP, C.W. et al. Effect of calibration on dispersion of glycohemoglobin values as determined by I I I laboratories using 21 methods. Clin Chem, v. 40, p. I 38-44, 1994.

31.WEYKAMP, C.W. et al. Carbamylated hemoglobin interference in glycohemoglobin assays. Clin Chem, v. 45, p. 438-40, 1999.

32. POSICIONAMENTO OFICIAL 2004. A importância da hemoglobina glicada (A/C) para a avaliação do controle glicêmico em pacientes com diabetes mellitus: aspectos clínicos e laboratoriais. Disponível em:< http://www.sbpc.org.br> Acesso em março 2006.

33. CAMARGO, J. L., GROSS, J. L. Conditions associated with very low values of glycohemoglobin measured by an HPLC method. J Clin Pathol, v. 57, p. 346-9, 2004.

\begin{tabular}{l|l} 
& Endereço para correspondência \\
\hline Andreza Fabro de Bem \\
Universidade Federal de Santa Maria \\
Centro de Ciências da Saúde \\
Departamento de Análises Clínicas e Toxicológicas \\
Faixa de Camobi, km 9 \\
CEP 97105-900 - Santa Maria-RS \\
Tel.: (55) 3220-8464 \\
e-mail: debemandreza@yahoo.com.br
\end{tabular}

